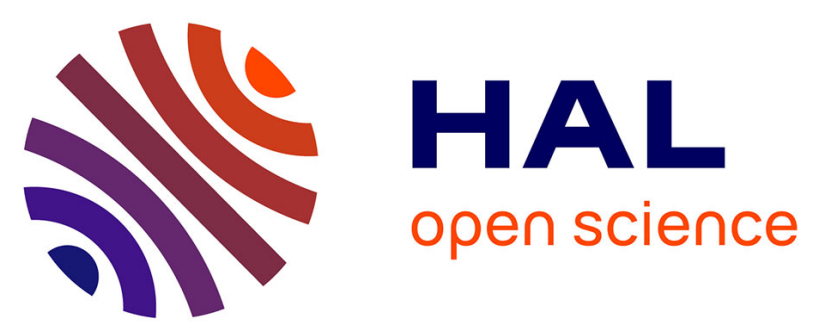

\title{
Innovative route for the preparation of high-performance polyolefin materials based on unique dendrimeric silica particles
}

Duarte Cecílio, Auguste Fernandes, João Paulo Lourenço, Timothy Mckenna, M. Rosário Ribeiro

\section{To cite this version:}

Duarte Cecílio, Auguste Fernandes, João Paulo Lourenço, Timothy Mckenna, M. Rosário Ribeiro. Innovative route for the preparation of high-performance polyolefin materials based on unique dendrimeric silica particles. Polymer Chemistry, In press, 10.1039/D1PY00453K . hal-03312466

\author{
HAL Id: hal-03312466 \\ https://hal.science/hal-03312466
}

Submitted on 5 Oct 2021

HAL is a multi-disciplinary open access archive for the deposit and dissemination of scientific research documents, whether they are published or not. The documents may come from teaching and research institutions in France or abroad, or from public or private research centers.
L'archive ouverte pluridisciplinaire HAL, est destinée au dépôt et à la diffusion de documents scientifiques de niveau recherche, publiés ou non, émanant des établissements d'enseignement et de recherche français ou étrangers, des laboratoires publics ou privés. 
Innovative route for the preparation of high-performance polyolefin materials based on unique dendrimeric silica particles

Duarte M. Cecílio ${ }^{[a]}$, Auguste Fernandes ${ }^{[a]}$, João Paulo Lourençco ${ }^{[a, b]}$, Timothy F.L. McKenna $^{[c]}$ and M. Rosário Ribeiro*[a]

[a] Centro de Química Estrutural (CQE), Departamento de Engenharia Química, Instituto Superior Técnico, Universidade de Lisboa, Av. Rovisco Pais 1, 1049-001 Lisboa, Portugal

${ }^{[b]}$ Departamento de Química e Farmácia, Faculdade de Ciências e Tecnologia, Universidade do Algarve, Campus de Gambelas, 8005-139 Faro, Portugal

${ }^{[c]}$ Université de Lyon, CPE Lyon, CNRS, UMR 5128 - Catalyse, Polymérisation, Procédés et Matériaux (CP2M), Bat 308F, 43 Blvd du 11 Novembre 1918, 69616 Villeurbanne, France

\section{Abstract}

In this study, an innovative methodology for the preparation of high-performance polyolefin-based materials combining a unique dendrimeric silica (DS) catalyst carrier, a straightforward in-situ metallocene catalyst supporting methodology and in situ ethylene polymerization technique was developed. This route combines metallocene supporting and polymerization in a single stage and avoids time-consuming and costly immobilization steps, allowing for a much more simplified experimental set-up. The impact of the immobilization procedure both on the catalytic activity for ethylene polymerization and on the morphological and thermal features of the ensuing polymers was investigated. The in-situ supporting procedure was shown to yield highly active catalysts, compared to a common approach involving a two-step immobilization procedure, and in the same order of magnitude of the reference molecular catalyst in homogeneous conditions. Moreover, the in-situ supporting route makes unnecessary the addition of external methyaluminoxane (MAO) cocatalyst thus, enabling a strong reduction of the MAO amount and potentially resulting in significant process cost savings. Moreover, polyethylene based materials with tunable molar masses, and desirable morphology and crystalline features were prepared, proving the method's versatility and ability in tailoring polymer properties, by changing the experimental conditions, and highlighting the potential of this methodology for the generation of highly performant HDPE nanocomposite materials for several applications. 


\section{Introduction}

The preparation of novel materials based on polymers has been a subject of much research in recent decades. Specifically, polyolefin/inorganic oxide nanocomposites show great interest as materials that exhibit reinforced mechanical, thermal and dielectric properties with regards to pristine polymers ${ }^{[1]}$. Preparation of these materials can be achieved by several processes, of which the main ones are blending and in-situ polymerization. The latter consists in the polymerization of the monomer around the pre-formed inorganic oxide reinforcement agent. As a technique, it presents the singular advantage of yielding a better dispersion of the filler nanoparticles, which results in a greater reinforcement effect ${ }^{[2,3]}$.

Metallocene catalysts for $\alpha$-olefin polymerization present themselves as interesting candidates as they are able to produce specialty grades of polyolefins with controlled polymer architecture and - therefore - properties. However, for them to be employed industrially in existing gas phase and slurry processes, they need to be supported on a solid material ${ }^{[4]}$. A wide variety of solids can be used for this purpose, from the more commonly chosen inorganic supports as is the case of silica ${ }^{[1]}$, alumina ${ }^{[5,6]}$, $\operatorname{zeolites}^{[7,8]}$, clays $^{[9,10]}$, magnesium chloride ${ }^{[11,12]}$ or even polymeric MAO, ${ }^{[13]}$ to organic supports such as $\operatorname{starch}^{[14]}$, cyclodextrin ${ }^{[15,16]}$ and synthetic polymers ${ }^{[17,18]}$. When considering commodity polyolefins, silica is perhaps the most commonly employed support. Ordered mesoporous silicas are also very attractive materials as supports for metallocene catalysts. They present organized mesopores, acting as nanoreactors that influence the rate and pattern of monomer insertion while simultaneously creating a confinement effect that promotes the blending of polymer chains at a nanometric scale ${ }^{[1,19]}$ and affects the crystallization of the macromolecules in its pores ${ }^{[20,21]}$. Moreover, the application of mesoporous nano-sized silicas for the production of polyolefin nanocomposites is of great interest as, contrary to micro-sized silica, the increase in interfacial area derived from the smaller nanoparticles leads to greater property improvement at the same filler loadings ${ }^{[22]}$.

In recent years, Polshettiwar et al. ${ }^{[23]}$ reported the discovery of a novel kind of fibrous nano-silica consisting of nanospheres with radial growth of dendrimeric fibers (KCC-1) that reportedly presents both high accessibility and high values of surface area. Its unique porosity and morphology create good accessibility, allowing for the application of fibrous nano-silica as an interesting inorganic support in catalysis as well as a filler agent in nanocomposite preparation. To the best of our knowledge, only two examples of fibrous silica-supported metallocene catalysts for ethylene polymerization are reported in literature ${ }^{[24,25]}$. Lee et al. ${ }^{[24]}$ 
employed KCC-1 as a support for a $\mathrm{Zr}$ metallocene catalyst in ethylene polymerization. The authors compared the novel dendrimeric silica nanospheres to industrially applied micronsized silica particles as support and observed an increase in activity from double to triple the activity exhibited by the micron-sized silica system when using KCC-1. In a more recent publication ${ }^{[25]}$, our group reported the use of similar dendrimeric silica nanospheres (DS) and aluminum-modified nanospheres (DSAl) prepared via different procedures as supports for $\mathrm{Cp}_{2} \mathrm{ZrCl}_{2}$ in ethylene polymerization. It was observed that different procedures for the preparation of DSAl lead to different textural and acidic properties of the resulting support. Moreover, the DSAI supports presented a unique behavior in catalysis, highlighting the effect of surface acidity and textural properties on the activation of the metallocene catalyst.

Regardless of the type of support chosen, the heterogenization of metallocene catalysts is a complex and time-demanding procedure which has significant economic impact and usually results in a system which is less active than its homogeneous analogue. The main routes used for the preparation of supported olefin polymerization catalysts are reported in a review article $^{[1]}$ focusing on the application of nanostructured silica materials in olefin polymerization. These routes may either involve impregnation techniques or covalent tethering of the catalyst or cocatalyst. Among impregnation techniques two main approaches are widely used: a direct impregnation procedure, where the metallocene contacts directly with the support, and a two-step impregnation procedure, which involves first the treatment of the support with MAO followed by contact with the metallocene. The increased number of steps required to immobilize the catalyst by covalent tethering may be considered economically unfavorable and precludes the wide use of this route.

A different and straightforward procedure, named in-situ supporting metallocene catalysts is based on a commercially available immobilized cocatalyst, SMAO (silica supported methylaluminoxane), and was reported by Chu et al. ${ }^{[4,26]}$ The procedure consisted in the direct addition of a metallocene solution to the silica-supported MAO present inside the reactor immediately prior to the monomer pressurization. This method renders the further addition of external MAO unnecessary, affording interesting activities at low to moderate $\mathrm{Al} / \mathrm{Zr}$ ratios. ${ }^{[26]}$. Another advantage of this procedure consists in avoiding time-consuming and costly steps, as well as yielding polymer with good morphology. Moreover, no reactor fouling is observed. Additionally, the in-situ supported system is more active than the standard supported one, while remaining less active than the homogeneous metallocene system. A subsequent report by Ahmadjo et al. ${ }^{[27]}$ demonstrated the importance of the support surface area on the performance of the catalytic systems prepared by this method. 
In this work we explore the use of the new dendrimeric silica (DS) as a catalyst carrier for coordination ethylene polymerization and aiming its further application for the production of high-performance polyethylene nanocomposites by in situ polymerization, where DS will also play the role of filler. It is well known that polymer nanocomposites properties depend on interfacial contact/adhesion between the two dissimilar organic and inorganic phases and consequently on the generated filler dispersion. Changes in catalyst preparation routes and polymerization procedures may result in a higher or lower degree of particle aggregation and therefore lead to differences in the filler dispersion of the issuing nanocomposites. Larger contact areas at interfaces will contribute to the enhancement of the mechanical response of the resulting materials. Thus, in a first investigation the impact of different methodologies for catalyst preparation and polymerization on the catalytic activity is addressed. In a subsequent phase the evaluation of the final properties of these materials and its correlation with the synthetic procedures will be carried out. With this goal in mind two different immobilization methodologies were implemented. One is inspired in the in-situ supporting metallocene catalysts procedure reported by Chu et al. ${ }^{[4]}$ and the other uses a more common route involving a two-step impregnation procedure. The results shown here demonstrate the improved catalytic behavior exhibited by this in situ supported catalytic system, as compared with the more conventional metallocene supporting procedures. The thermal and morphological features of the polymers obtained are also evaluated. These results will be fundamental for the ongoing work concerning the impact of different in situ polymerization procedures on the properties of the ensuing nanocomposites.

\section{Experimental Section}

Materials For the synthesis of the DS materials, tetraethyl orthosilicate (TEOS, 99\%, Aldrich was used as a silicon precursor. 1-hexadecylpyridinium bromide hydrate (CPB, 98\%, Alfa Aesar), cyclohexane (99.4\%, Chem-Labs) and 1-pentanol (99.4\%, VWR) were used as template, solvent and co-solvent, respectively. For the preparation of the solid activator, methylaluminoxane (PMAO-IP 7wt\% in toluene, Akzo Nobel) was used as co-catalyst. Regarding the ethylene polymerization reactions, zirconocene dichloride $\left(\mathrm{Cp}_{2} \mathrm{ZrCl}_{2}, \mathrm{Cp}=\eta^{5}-\mathrm{C}_{5} \mathrm{H}_{5}\right.$, Aldrich) was used as metallocene catalyst and triisobutylaluminum (TIBA, [( $\left.\left.\mathrm{CH}_{3}\right)_{2} \mathrm{CHCH}_{2}\right]_{3} \mathrm{Al}$, Aldrich) was used as a scavenger. Ethylene and nitrogen (Air Liquide) were purified through adsorption columns containing a mixture of $4 \mathrm{~A}$ and $13 \mathrm{X}$ molecular sieves. Toluene (VWR) was dried by refluxing over metallic sodium under nitrogen and using benzophenone as an indicator. Other materials were used without further purification. All sensitive reactants and materials were handled under nitrogen using standard inert atmosphere techniques. 
Dendrimeric silica nanospheres (DS) synthesis and characterization: The synthesis procedure used was described in a previous work ${ }^{[25]}$. CPB (3g, $\left.0.0078 \mathrm{~mol}\right)$ and urea $(1.8 \mathrm{~g}, 0.03$ $\mathrm{mol})$ are dissolved in deionized water $(90 \mathrm{~mL})$. Separately, pentanol $(1.5 \mathrm{~mL})$ and TEOS $(7.5 \mathrm{~g}$, $0.036 \mathrm{~mol}$ ) are dissolved in cyclohexane $(90 \mathrm{~mL})$. The two solutions are stirred separately to ensure homogenization. The organic solution is added to the aqueous solution under vigorous stirring and the resulting mixture is stirred for 30 minutes at room temperature. The microemulsion formed is finally treated at $120^{\circ} \mathrm{C}$ with microwave (MW) radiation for 60 minutes in Teflon autoclave vessels (MARS-5 oven, $600 \mathrm{~W}$ maximum power). The DS material is separated by centrifugation and washed twice with a 1:1 water and acetone solution. The resulting material is air-dried for 24 hours and after calcined at $650{ }^{\circ} \mathrm{C}$ under air for 8 hours. The characterization of the support was performed as describred in a previous publication ${ }^{[25]}$, with regards to nitrogen sorption measurements and morphology assessment through transmission electron microscopy.

Support Pretreatment: A batch of DS was treated at $200^{\circ} \mathrm{C}$ under primary vacuum for 90 minutes to remove the adsorbed water.

Pre-treatment of the support with MAO followed by immobilization of the zirconocene (DS-MAO method): Approximately $500 \mathrm{mg}$ of dry support were weighed and stored in a degassed Schlenk tube. Toluene was added with a ratio of $25 \mathrm{~mL}$ per gram of support and vigorously stirred. MAO was added to the suspension to achieve a surface Al loading of $4 \mathrm{mmol} / \mathrm{g}$ and vigorously stirred for 16 hours at room temperature, shielded from ambient light. Afterwards, the toluene was removed under vacuum until a light and dry powder was obtained. The dry powder was re-suspended in toluene with a ratio of $29 \mathrm{~mL}$ per gram of support and a toluene solution of zirconocene dichloride was prepared and added to achieve a final $\mathrm{Zr}$ loading of $35 \mu \mathrm{mol}$ per gram of support. After a contact time of 4 hours, the final catalyst was stored in the Schlenk tube. The supernatant or clarified liquid test was followed as described in the literature for the DS-MAO method, in order to assess wether the catalyst was totally imobilized on the support surface ${ }^{[28]}$. The sample was initially named according to the method, so as to compare against the remaining procedures, and subsequently named DS-MAO-Al/ZrX, where $\mathbf{X}$ stands for the total $\mathrm{Al} / \mathrm{Zr}$ ratio (both supported and external MAO) in the reaction medium.

Pretreatment of the support with MAO for the preparation of a supported activator (SA-DS method): Approximately $500 \mathrm{mg}$ of dry support were weighed and stored in a degassed Schlenk tube. Toluene was added with a ratio of $25 \mathrm{~mL}$ per gram of support and vigorously 
stirred. MAO was added to the suspension to achieve a surface Al loading of $3 \mathrm{mmol} / \mathrm{g}$ and vigorously stirred for 16 hours at room temperature, shielded from ambient light. The suspension is stored in a Schlenk tube and directly used in the polymerization procedure. The sample was initially named according to the method, so as to compare against the remaining procedures, and subsequently named SA-DS-AI/ZrX, where $\mathbf{X}$ stands for the ratio of supported MAO activator $\mathrm{Al}$ to $\mathrm{Zr}$ present in the reaction medium.

Ethylene Polymerization The polymerization reactor consists of a $250 \mathrm{~mL}$ bottle (Wilmad LabGlass LG-3921), with crown cap, gasket and magnetic stirrer. This reactor was placed in a water bath. Ethylene consumption rate was measured using two mass flow controllers (Hastings Instruments HFC-202 and Alicat Scientific 16 Series) and recorded in a personal computer with data acquisition hardware and software (a ComputerBoards CIO-DAS08/Jr-AO interface card with Labtech DataLab software). Ethylene pressure was measured with a digital manometer (Air Liquide M2500) and also recorded.

The reactor was purged with vacuum/ $\mathrm{N}_{2}$ and loaded with enough toluene to match a total volume of $50 \mathrm{~mL}$ when the polymerization was started. Nitrogen was then replaced with ethylene by means of 5 vacuum/ethylene cycles and afterwards waiting for the ethylene consumption to stabilize. At this point, the procedure changes according to the preparation method employed to prepare the catalytic system:

In the case of the SA-DS method, there is an initial addition of $1.0 \mathrm{~mL}$ of a $0.1 \mathrm{M}$ TIBA solution to act as scavenger, followed by the addition of the appropriate volume of DSsupported MAO activator and finally the addition of a $\mathrm{Cp}_{2} \mathrm{ZrCl}_{2}$ toluene solution corresponding to $1.9 \times 10^{-6} \mathrm{~mol}$ of $\mathrm{Zr}$. It is worth noting that no external MAO solution is added in this procedure and the TIBA alkylaluminum compound is added in reduced amounts compared to the supported activator to yield solely a scavenging role.

The procedure involving the DS-MAO method consists in the addition of the appropriate amount of external MAO solution to achieve an $\mathrm{Al} / \mathrm{Zr}$ ratio of 1500 in the polymerization medium, followed by the addition of a vigorously stirred suspension containing the zirconocene catalyst supported onto MAO pretreated DS, equivalent to $1.8 \mu \mathrm{mol}$ of $\mathrm{Zr}$.

In the reference polymerization with the zirconocene in homogeneous conditions, an amount of MAO solution is added to achieve an $\mathrm{Al} / \mathrm{Zr}$ ratio of 1500 in the polymerization medium, followed by the addition of a $\mathrm{Cp}_{2} \mathrm{ZrCl}_{2}$ solution in toluene, equivalent to $1.9 \mu \mathrm{mol}$ of Zr. 
The polymerizations took place at $25^{\circ} \mathrm{C}$ and 1.1 bar of ethylene. During the reaction the temperature, pressure and ethylene mass flow data are monitored in real-time and automatically recorded. The ethylene mass flow was converted to ethylene consumption and polymerization activity calculated in $\mathrm{kg}_{\mathrm{PE}} / \mathrm{mol}_{\mathrm{Zr}} \mathrm{h}$. The kinetic profiles correspond to ethylene consumption versus time, which after integration yields a value of average activity that was compared to the value obtained by considering the mass of recovered polymer. The reaction time was controlled to achieve a desired polymer amount through the measurement of ethylene consumption at the mass flowmeters. After the reaction, possible solubilized polymer is precipitated over $5 \% \mathrm{HCl}$ acidified methanol, filtered and washed twice using methanol before drying.

Polymer molar mass characterization: Polymer average molar mass and dispersity were obtained through high temperature size exclusion chromatography (HT-SEC) measurements, employing a Malvern Instruments Viscotek system outfitted with three Polefin $300 \mathrm{~mm} \times 8 \mathrm{~mm}$ I.D. columns from Polymer Standards Service with corresponding porosities of $100 \AA, 100,000 \AA$ and 1,000,000 ̊.. 1,2,4-trichlorobenzene (TCB) was used as an eluent at a 1 $\mathrm{ml} \mathrm{min}{ }^{-1}$ flowrate and a temperature of $150{ }^{\circ} \mathrm{C}$. 2,6-di(tert-butyl)-4-methylphenol was used as an eluent stabilizer under a $200 \mathrm{mgL}^{-1}$ concentration. The polymers were dissolved in TCB at an approximate concentration of $1 \mathrm{mgmL}^{-1}$ and $200 \mu \mathrm{L}$ injections were performed. Online detection was performed by means of a differential refractive index detector, a viscosity detector and a dual light scattering detector (RALS and LALS) for accurate measurements. OmniSEC version 5.02 was used to calculate the polymer properties.

Scanning Electron Microscopy (SEM) characterization of polymer morphology: SEM micrographs were obtained on a JEOL JSM-7001F equipment coupled with an Oxford EDX detector. Powders prepared from ethylene polymerization were deposited in a $\mathrm{Cu} /$ polymer grid sample holder to analyze their morphological characteristics.

\section{Calorimetric characterization of polymer crystallinity and melting transitions:} Calorimetric analyses were carried out in a Mettler Toledo DSC 3+ model calorimeter connected to a cooling system. Samples weighed approximately $5 \mathrm{mg}$. A temperature interval from $-40{ }^{\circ} \mathrm{C}$ to $160^{\circ} \mathrm{C}$ was chosen and a $10^{\circ} \mathrm{C} / \mathrm{min}$ heating rate was employed. A $290 \mathrm{~J} / \mathrm{g}$ value was used for the enthalpy of fusion of a perfectly crystalline polyethylene ${ }^{[29,30]}$. 


\section{Results and Discussion}

The synthesis of a reference batch of DS was successful and its characterization was detailed in a previous publication ${ }^{[25]}$. The morphology of the DS nanospheres is highlighted in Figure 1. The TEM obtained micrograph confirms the dendrimer-like morphology of the silica, highlighting its particular porosity created by the free space between the radially growing fibers. Regarding the particle size distribution, the nanospheres present particle diameters ranging from 100 to $600 \mathrm{~nm}$. Nitrogen sorption measurements indicated a BET surface area of $370 \mathrm{~m}^{2} / \mathrm{g}$ and a porous volume $0.7 \mathrm{~cm}^{3} / \mathrm{g}$.
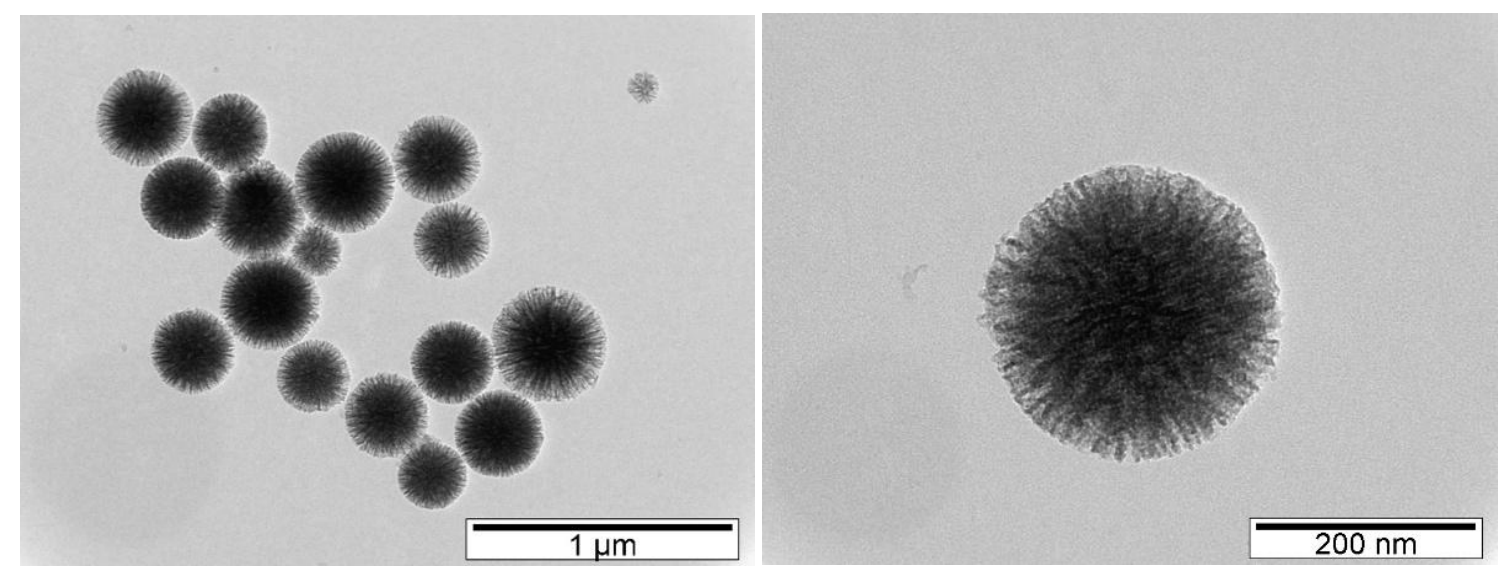

Figure 1 - TEM micrograph of the DS nanospheres used as a catalytic support at two different magnifications.

As previously mentioned, two different methodologies were employed for the preparation and evaluation of the activity exhibited by the supported catalyst systems. The first, named DS-MAO, uses a common approach consisting in a two-step immobilization procedure (the support is first treated with MAO and subsequently impregnated with the zirconocene catalyst precursor, following a protocol described in previous studies of our group $\left.^{[21,31,32]}\right)$. The polymerization of ethylene is then promoted by this supported catalyst in presence of an additional amount of MAO. The second one, SA-DS, is a straightforward route inspired in a few literature studies by Chu et al. ${ }^{[4,26]}$, reporting the use of an in-situ supported catalyst, obtained by contacting a commercial silica supported MAO activator with a homogeneous metallocene solution directly in the polymerization reactor. The same concept is used here in order to prepare a DS supported MAO activator. This route combines metallocene supporting and polymerization in a single step, and allows for a much more simplified experimental set-up, with no need for an additional catalyst impregnation step prior to the reaction. Another advantage is that this in-situ formed catalyst is active in the absence of additional MAO and only requires the use of a small amount of an alkylaluminum during 
ethylene polymerization, to act as impurity scavenger and/or alkylating agent. The differences in the conditions used for the two methodologies arise directly from the distinctive features and constraints of each one of the procedures selected.

The data presented in Table 1 shows a summary of the polymerization conditions and the activity, polymer molar mass and dispersity for the two methodologies used. For comparison purposes, results obtained for ethylene polymerization with the homogeneous catalyst are also shown.

Table 1 -Polymerization conditions, activities and molar masses obtained for the polymers prepared via the two different methods compared to polyethylene prepared with the homogeneous catalyst.

\begin{tabular}{|c|c|c|c|c|c|c|c|}
\hline Sample & $\begin{array}{l}\text { Al loading } \\
\text { on support } \\
\text { (mmol/g) }\end{array}$ & $\begin{array}{c}\text { Zr loading } \\
\text { on } \\
\text { support } \\
\text { ( } \mu \mathrm{mol} / \mathrm{g} \text { ) }\end{array}$ & $\begin{array}{c}\mathrm{Zr} \\
\text { loading } \\
\text { in } \\
\text { reactor } \\
\text { ( } \mu \mathrm{mol})\end{array}$ & $\begin{array}{l}\mathrm{Al} / \mathrm{Zr} \text { in } \\
\text { reactor }\end{array}$ & $\begin{array}{l}\text { Average Activity } \\
\left(\mathrm{kg}_{\mathrm{PE}} / \mathrm{mol}_{\mathrm{Zr}} \mathrm{h}\right)^{[\mathrm{a}]}\end{array}$ & $\begin{array}{c}M_{w} \\
(\mathrm{~g} / \mathrm{mol})\end{array}$ & $M_{w} / M_{n}$ \\
\hline $\mathrm{HDPE}^{[\mathrm{b}]}$ & - & - & 1.9 & 1500 & 7070 & 221000 & 2.3 \\
\hline DS-MAO ${ }^{[c]}$ & 4 & 35 & 1.8 & ca. 1600 & 3530 & 515000 & 2.0 \\
\hline SA-DS ${ }^{[\mathrm{d}]}$ & 3 & - & 1.9 & ca. 160 & 8300 & 281000 & 2.4 \\
\hline
\end{tabular}

${ }^{\text {[a] }}$ Presented for 3 minutes of polymerization time for the SA-DS procedure and 5 minutes for the remaining samples.

${ }^{[b]}$ Produced with the $\mathrm{Cp}_{2} \mathrm{ZrCl}_{2}$ metallocene in homogeneous conditions

[c] Produced employing the DS-MAO methodology

${ }^{\text {[d] }}$ Produced employing the SA-DS methodology

The activity results presented in Table 1 immediately highlight a significant and clear difference between the different methods, in particular between the more commonly applied DS-MAO route and the others. Considering the homogeneous system as a reference, we can see that the DS-MAO route yields a lower catalytic activity. This is a typical behavior of many supported catalysts prepared in this conventional procedure (immobilization of the metallocene in a MAO pretreated support). The steric hindrance provided by the support, that acts as an extremely bulky ligand, combined with an inefficient generation of active sites or even the deactivation of active sites that are effectively generated, are among the aspects that may influence the behavior herein observed ${ }^{[33-35]}$. Nevertheless, it is worth highlighting that the DS-MAO system achieves half the average activity yielded by the homogeneous metallocene for the same polymerization time and similar $\mathrm{Al} / \mathrm{Zr}$ ratio.

On the other hand, the SA-DS methodology leads to a high activity value compared to the DS-MAO methodology, and in the same order of magnitude of the reference homogeneous catalyst system. This is an interesting result, as it is achieved using a much lower amount of MAO activator than the other two procedures ( $\mathrm{Al} / \mathrm{Zr}=160$ instead of $1500-1600)$. 
In order to get further insight to the behaviour of these different catalytic systems their kinetic profiles are compared in Figure 2.

Figure 2 shows two plots, the left one depicting the kinetic profiles comparing catalyst systems prepared via the SA method, the DS-MAO method and a reference polymerization profile done with the metallocene in solution (homogeneous conditions), and the right one depicting the respective kinetic profiles normalized against the activity maximum, which allows for an easier comparison of the relative polymerization rate decay for these systems and, consequently, of the relative importance of catalyst deactivation and/or possibly occurring external diffusion limitation phenomena associated in each case. Moreover, as the final goal is the preparation of nanocomposites, the polymerization time was controlled in each case in order to achieve a desired composition of the final material in terms of mass of polymer and mass of support/inorganic filler.
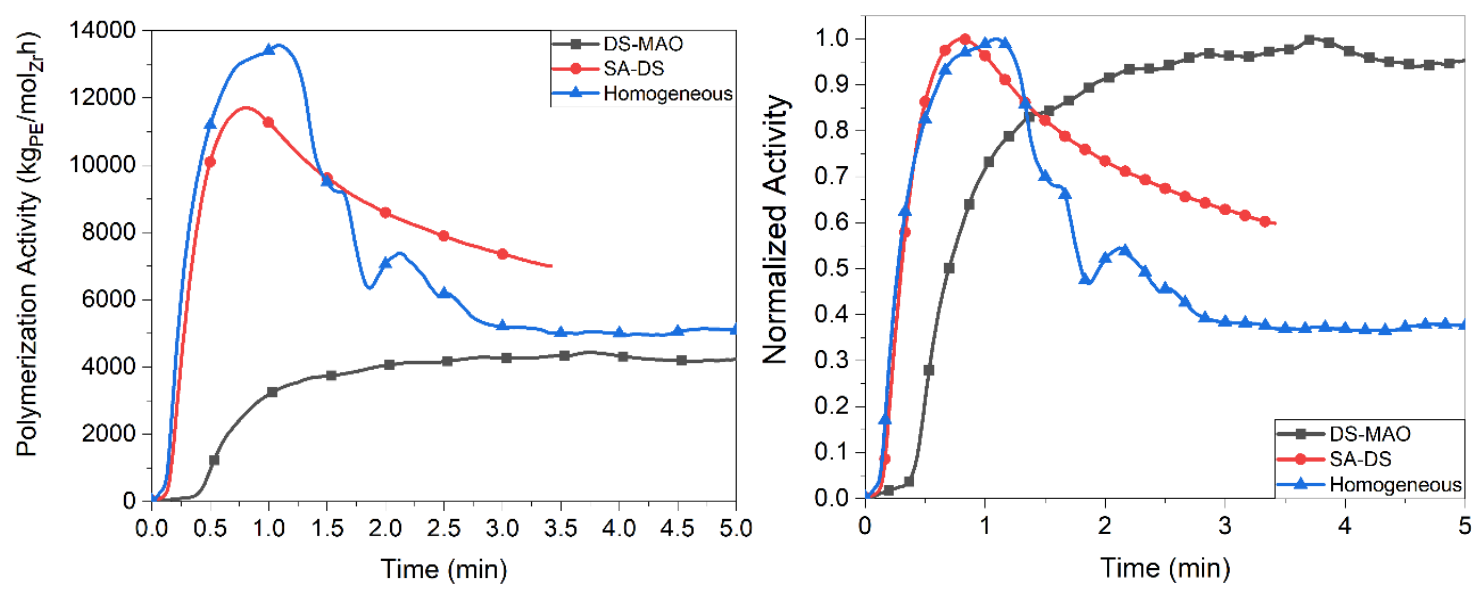

Figure 2 - Kinetic profiles (left) and normalized kinetic profiles (right) for the catalyst system prepared via different methods in ethylene polymerization.

The difference between the kinetic behavior of the different samples is clearly seen. The homogeneous reference profile presents a typical pattern where the activity peak is followed by a steady decline until a plateau is reached, the value of which is not much higher than the stable plateau reached for the DS-MAO kinetic profile. This may be ascribed to catalyst deactivation by bimolecular routes and/or to the very high activities exhibited by the homogeneous systems which promptly consume all available ethylene dissolved in the reaction medium and - as such - although the catalyst deactivation is the most probable cause for the observed behavior, the possible occurrence of external diffusion limitations cannot be discarded.

On the other hand, a build-up profile without rate decay is observed for DS-MAO methodology. The combined effect of the support and of the MAO (especially at the high 
$\mathrm{Al} /$ Metal ratio high used) on decreasing metallocene bimolecular deactivation and on the stabilization of the cationic metallocene alkyl species is expected to contribute to active site stabilization, leading to a non-decay type profile. ${ }^{[36]}$

In the case of SA-DS route, the very low Al/Zr ratio used in this method (160) may not provide an effective stabilization of the cationic metallocene alkyl active species, thus also contributing to the observed decay type kinetic profile due to the aforementioned role of $\mathrm{MAO}^{[37]}$. Nevertheless, an increase in catalyst stability may be observed for the SA-DS method in comparison to the homogeneous catalyst when looking to the profiles of the normalized activity (see Figure 2, right plot).

As mentioned before, contrary to the DS-MAO method, the SA-DS one does not include the addition of external MAO to the reaction medium, relying instead on the addition of a very small amount of TIBA to scavenge impurities in the medium. As such, the high activities observed are achieved with a significantly lower excess of MAO, compared to the two other methods. The available data on the use and kinetics of this type of in-situ supported catalysts for ethylene polymerization is very scarce. However, Chu and co-workers ${ }^{[4]}$ reported the use of an in-situ supported catalyst for ethylene polymerization (at 80 psig and $60 \circ \mathrm{C}$ ), prepared in the polymerization reactor by the simultaneous addition of a homogeneous solution of $\mathrm{Et}[\mathrm{Ind}]_{2} \mathrm{ZrCl}_{2}$, a commercial MAO supported on silica, (SMAO, supplied from Witco; Al content $=24.4 \mathrm{wt} \%$ ), and trimethylaluminum (TMA). Contrary to our results, the polymerization rate profiles of the in-situ supported metallocene catalysts did not show rate decay as a function of time. A model for the polymerization mechanism of in-situ supported metallocene catalysts was proposed by the authors. The observed behavior was attributed to the reactivation - or substitution - of deactivated species on the SMAO surface by complexation with the solubilized metallocene (and possibly with TMA leading to a dynamic equilibrium). Further evidence apparently supporting this hypothesis was provided by subsequent investigations with these in-situ supported systems at a constant metallocene amount and varying amounts of the commercial SMAO ${ }^{[26]}$. At lower $\mathrm{Al} / \mathrm{Zr}$ ratios (where the probability of having unsupported metallocene species was higher), no polymerization rate decay was found while at higher $\mathrm{Al} / \mathrm{Zr}$ ratios the ethylene feed rate decreased with time. The much lower amount of metallocene ( $\sim 3$ times) used in the present study, together with the results of a polymerization test with the solvent supernatant showing no activity, seem to indicate that no significant soluble metallocene species are present. This way, and according to the mechanism proposed by Chu et al., the negligible amount of soluble metallocene could be one factor contributing to the decay type kinetics observed. 
Ahmadjo et al. ${ }^{[27]}$ used in-situ impregnation method to immobilize Bis(2- $\mathrm{R}$ - ind) $\mathrm{ZrCl}_{2}$ ( $\mathrm{R}$ : $\mathrm{H}$ or Phenyl) on different types of silica and investigated the effect of support type on the performance of the immobilized catalyst in ethylene polymerization. The authors have found that the kinetic profiles obtained were dependent on the surface area. For PQ-3060 support, with a surface area of $570 \mathrm{~m}^{2} / \mathrm{g}$ the kinetic curves showed a decay type which was shifted to stable type when using MCM-41 as support, with a surface area of $1100 \mathrm{~m}^{2} / \mathrm{g}$. The higher stability of the kinetic profile of the catalyst supported on MCM-41 was attributed to the better accessibility of the active centers during polymerization. As proposed before ${ }^{[4,38]}$, the authors also assume that in the in-situ supported catalysts new catalytic sites are continuously formed on the external surface of the silica particles by the adsorption of the catalyst species which are available in the liquid phase during polymerization. In previous publications by Sano et $a l .{ }^{[39]}$ and Kumkaew et $a I^{\left[{ }^{[0,41]}\right.}$ the key role of the pore volume of support on catalyst activity was shown, whereas the effect of surface area has not been taken into account. The crucial effect of the surface area of the support on the performance of the in-situ supported catalysts was attributed by Ahmadjo et al. ${ }^{[27]}$ to the decreased contact time during catalyst impregnation in this method, which amplifies the importance of the probability of the contact between catalyst compounds and the exterior potential impregnation sites. Thus, it is highly likely that the surface area of the support presents a decisive role in the in-situ impregnation method, unlike in more traditional techniques. Due to the particular morphology of our support, where a narrowing of the pores towards the particle center is expected to occur, and despite its moderate surface area, a significant part of the surface is expected to remain highly accessible and, consequently, a high catalytic activity was obtained.

Another very significant observation, when comparing our results with those from Chu et $a l^{[4]}$, is that while they found a reduction of activity for the in-situ supported system when compared to the soluble system, in our specific conditions a high polymerization activity is kept for the in-situ prepared catalyst (similar to the homogenous reference).

It is well known that conditions for catalyst preparation such as temperature, use of solvent and contact time affect the performance of the final system ${ }^{[42]}$. Moreover, the ethylene pressure and temperature of the polymerization reaction itself may also dramatically change the activity of the system, as well as the observable kinetic profile. As stated before, our procedure, although inspired on the in-situ supporting procedure used and reported by Chu et al. ${ }^{[4]}$, shows very significant changes both in terms of the supported activator features and of the polymerization conditions used for the in-situ supporting procedure. 
Firstly, the novel DS supported MAO activator developed in this study has a nanometric size and shows a unique dendrimer-like morphology and a lower MAO loading (8 wt. \%) when compared with the Al content of commercial SMAO (24 wt. \%), and this was an important factor identified, both in earlier ${ }^{[43]}$ and more recent studies ${ }^{[44]}$, as strongly affecting catalytic performance and even polymer properties. Additionally, the polymerization reaction is performed with a different metallocene and alkylaluminum $\left(\mathrm{Cp}_{2} \mathrm{ZrCl}_{2}\right.$ instead of $\mathrm{EtInd}_{2} \mathrm{ZrCl}_{2}$ and TIBA instead of TMA). Moreover, different polymerization conditions are also used, (lower temperature and ethylene pressure, hexane was replaced by toluene). Undoubtedly, all these aspects influence the observed activity and the developed procedure overall yields a high activity while using approximately $10 \%$ the amount of MAO of the alternative methods.

The HDPE produced through the DS-MAO method presents an extremely high $M_{w}$ value compared to that observed for the HDPE produced with the metallocene in homogeneous conditions, which suggests that the active species formed employing this method strongly benefits from the steric protection offered by the support, preventing $\beta$ hydride transfer. This behavior has previously been observed and attributed to the blocking of one of the sides of the zirconium active site by the support, which hinders $\beta$-hydrogen elimination transfer between two different transition metal centers and promotes the formation of larger polymer chains with accordingly higher average molar mass ${ }^{[45,46]}$. Brambilla et al. ${ }^{[47]}$ also observed similarly high $M_{w}$ values for a silica supported $\mathrm{Cp}_{2} \mathrm{ZrCl}_{2}$ system for ethylene polymerization, although the authors did not suggest further explanation for the high average molar mass value apart from the steric effect of the support. On the other hand, the polymer produced via the SA-DS method presents an $M_{w}$ value closer to that exhibited by the reference homogeneous HDPE, albeit slightly higher, suggesting that the active species is somewhat weakly bound to the support surface and that the support steric protection effect, although present, is not as effective as for the DS-MAO procedure.

The results presented so far show an interesting behavior for the SA-DS route and potential as a straightforward polymerization methodology. Several polymerization reactions were subsequently conducted, varying the amount of MAO introduced through the introduction of increasing amounts of supported activator, while keeping the metallocene catalyst amount unchanged. The effects on the kinetic profile, catalytic activity and polymer structure were studied. Results are shown in Figure 3 and Table 2. 

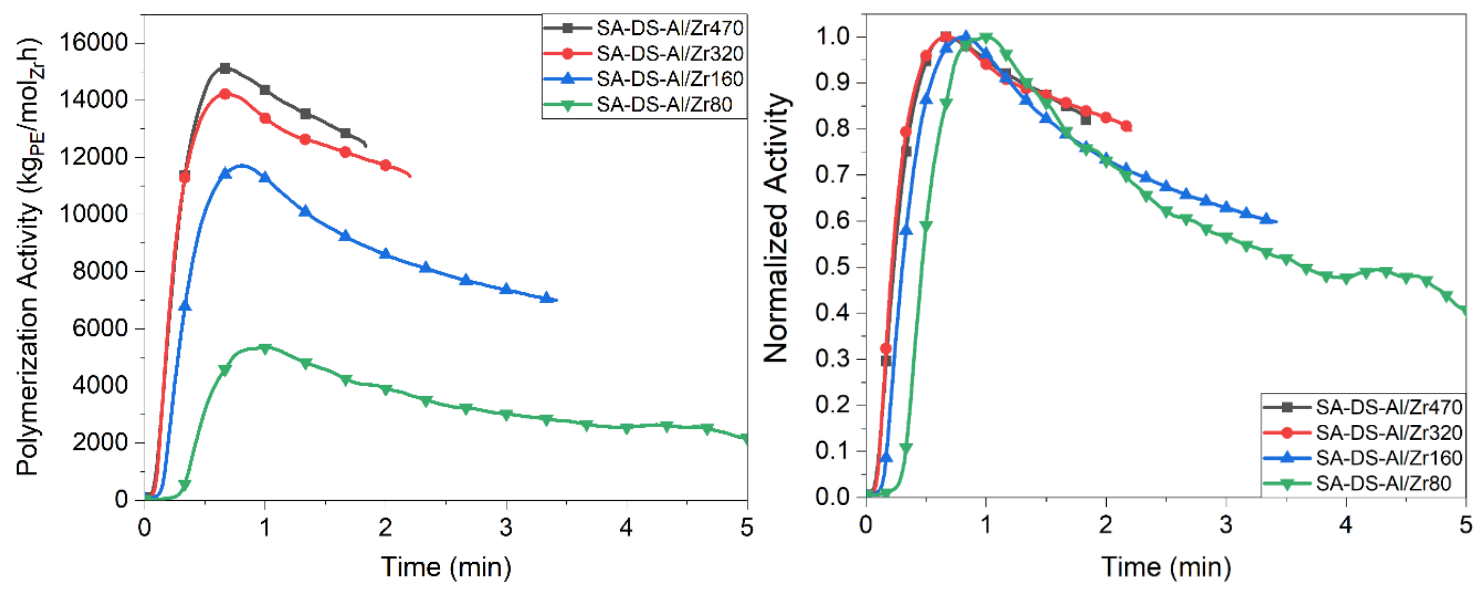

Figure 3 - Kinetic profiles (left) and normalized kinetic profiles (right) for the catalyst system prepared via the supported activator method (SA-DS) in ethylene polymerization with varying supported activator Al/Zr ratios.

Table 2 presents the conditions used in the polymerization reactions varying the Al/Zr ratio for the SA-DS methodology. The average polymerization activity, weight average molar mass $\left(M_{w}\right)$ and dispersity $\left(M_{w} / M_{n}\right)$ values are presented for each sample.

Table 2 - Polymerization conditions, activities and molar masses obtained for the nanocomposites prepared via the SA-DS method with varying Al/Zr ratios.

\begin{tabular}{|c|c|c|c|c|c|c|}
\hline Sample & $\begin{array}{c}\text { Al loading } \\
\text { on support } \\
\text { (mmol/g) }\end{array}$ & $\begin{array}{c}\text { Zr loading } \\
\text { in reactor } \\
(\mu \mathrm{mol})\end{array}$ & $\mathrm{Al} / \mathrm{Zr}$ & $\begin{array}{l}\text { Average Activity } \\
\left(\mathrm{kg}_{\mathrm{PE}} / \mathrm{mol}_{\mathrm{Zr}} \mathrm{h}\right)^{[\mathrm{a}]}\end{array}$ & $\begin{array}{c}M_{w} \\
\text { (g/mol) }\end{array}$ & $M_{W} / M_{n}$ \\
\hline SA-DS-AI/Zr470 & \multirow{4}{*}{3} & \multirow{4}{*}{1.9} & ca. 470 & 12130 & 224000 & 2.1 \\
\hline SA-DS-Al/Zr320 & & & ca. 320 & 11510 & 205000 & 2.7 \\
\hline SA-DS-Al/Zr160 ${ }^{[\mathrm{b}]}$ & & & ca. 160 & 8300 & 281000 & 2.4 \\
\hline SA-DS-AI/Zr80 & & & ca. 80 & 3640 & 423000 & 2.3 \\
\hline
\end{tabular}

[a] Presented for 3 minutes of polymerization time for all samples.

[b] Corresponds to sample SA-DS in Table 1

An analysis of the results in Table 1 and Table 2 supports the finding that the SA-DS method requires much lower excesses of MAO compared to the other methods in order to achieve high catalytic activity. In fact, even for the lowest $\mathrm{Al} / \mathrm{Zr}$ ratio of 80 , the average activity is comparable to that of the DS-MAO-AI/Zr1600 sample (see Table 1), although the presence of external MAO at high $\mathrm{Al} / \mathrm{Zr}$ ratios in the latter sample will enable active site stabilization, resulting in an increase of the average activity overtime (Figure 2), whereas it will decrease in the case of the SA-DS method samples (Figure 3). Additionally, the increase of the amount of 
activator in relatively small increments dramatically increases activity, as is presented in Table 2 , while maintaining the same type of kinetic profile. Although an increase in activity being associated with an increase in MAO amount has been widely reported in literature, this increase is usually associated with quite higher excesses of MAO, upwards of 1000 times the amount of transition metal ${ }^{[48]}$.

It is important to highlight that from an $\mathrm{Al} / \mathrm{Zr}$ of 320 or higher, a diminishing return is observed. That is, an increase in the $\mathrm{Al} / \mathrm{Zr}$ ratio for values higher than 320 presents a diminishing improvement in the observed polymerization activity. Sample SA-DS-AI/Zr-320 appears to present a good compromise between the amount of activator introduced and the activity exhibited by the system.

The right plot of Figure 3 shows the normalized kinetic profiles based on the left plot. This plot confirms some observations previously made while also adding interesting information on the relative polymerization rate decay under the distinct conditions used. It appears that an increased amount of supported activator leads to a decrease in deactivation rate, as shown by the more slowly deactivating curves for higher $\mathrm{Al} / \mathrm{Zr}$ ratios. This result may also be due to the presence of higher amounts of MAO and to its aforementioned role in the stabilization of generated active sites.

Analyzing the molar mass data presented in Table 2 , it is possible to see that, similarly to the other methods, SA-DS samples present $M_{w}$ and dispersity values in the range of metallocene HDPE, regardless of the $\mathrm{Al} / \mathrm{Zr}$ ratio of the system. It is also clear from the results that the weight average molar mass of the polymers decreases with the increase of the Al amount in the polymerization medium. In fact, the molar mass of the polymer almost doubles from the highest Al content to the lowest. The plot presented in Figure 4 confirms this trend, which may be related to an increase in chain transfer reactions to the aluminum. Considering that there are two competing reaction pathways consisting in chain propagation on the transition metal center and in transfer reaction to the Al present in the activator, if one were to increase the amount of $\mathrm{Al}$ in the medium it would shift the balance towards the chain transfer reaction due to the high availability of Al centers. In this case, we would have a much higher transmetalation reaction rate, causing the chains to shift between the $\mathrm{Zr}$ and Al metal centers often between monomer insertions and effectively resulting in shorter chains at the end of polymerization. ${ }^{[49]}$ 


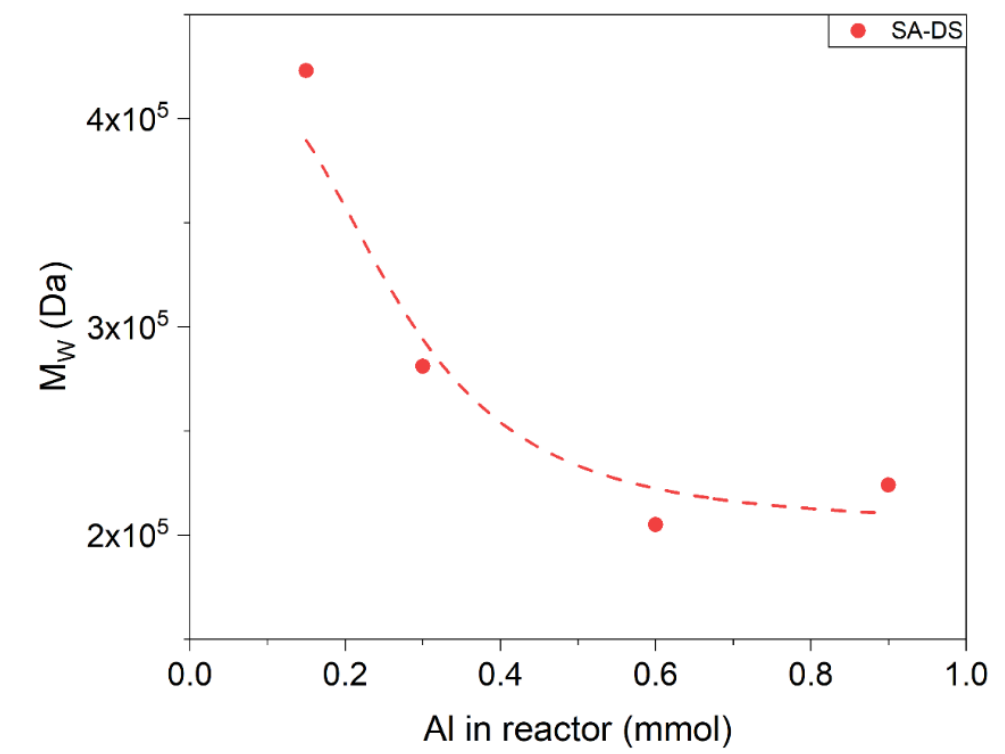

Figure 4 - Variation of SA-DS' molar mass with the Al content in the reactor during polymerization.

\section{Morphology of the reactor powders}

The nascent polymer powders recovered directly from the reactor were washed, air dried and then analyzed in a scanning electron microscope. These polymer powders were obtained by conducting extremely short polymerization reactions, at a total polymerization time of 30 seconds. The goal of these polymerization runs is to better understand the generation of polymer from the nanospheres. The results aimed to see if the different methods would yield significant differences in terms of morphology, which could eventually help understand other properties. Figure 5 and Figure 6 show the morphology of the polymers produced with the two different preparation methods.

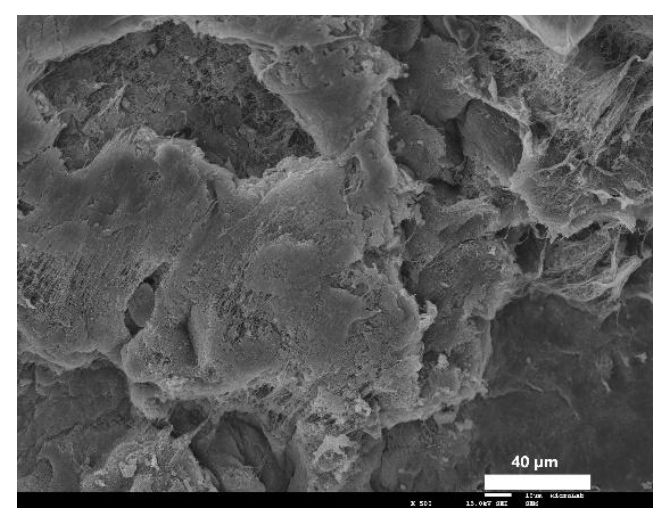

(a)

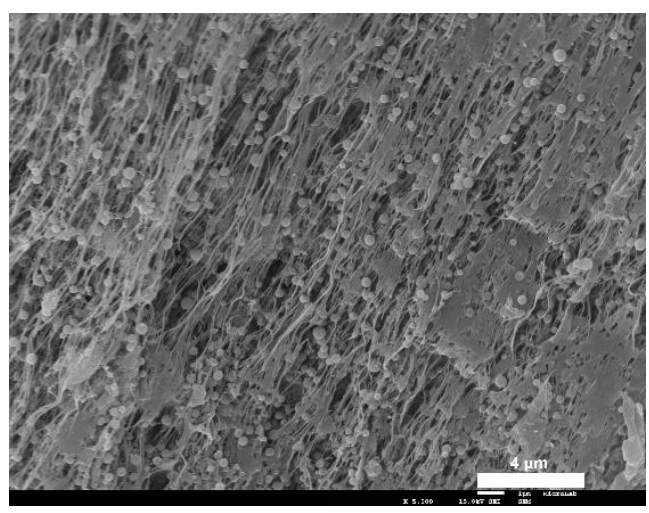

(b) 


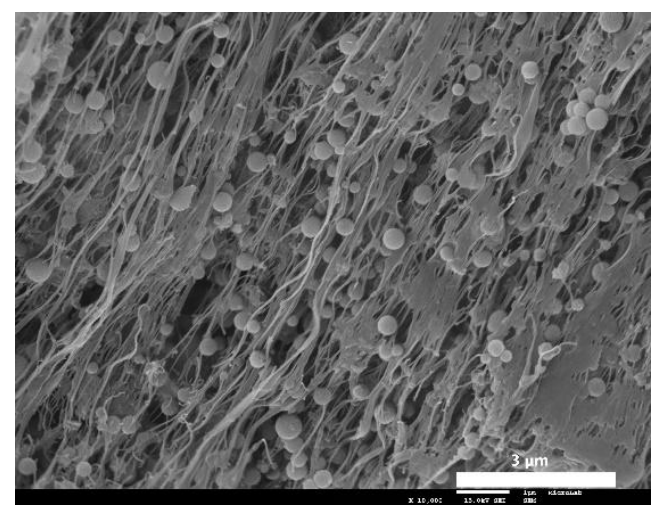

(c)

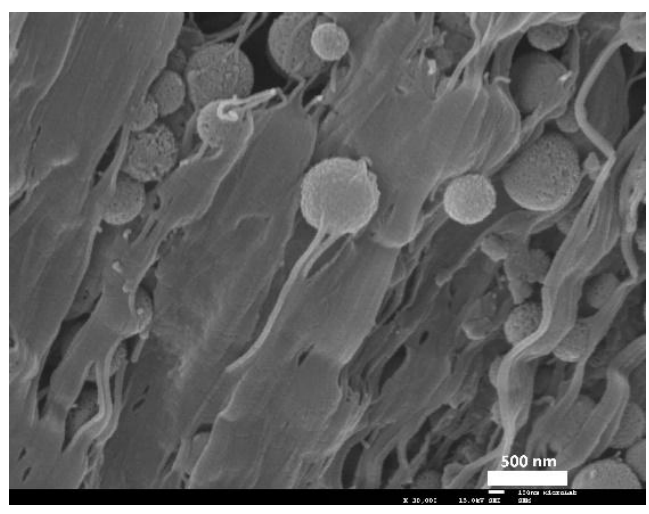

(d)

Figure 5 - SEM micrographs of polyethylene particles produced via the DS-MAO method with different magnifications: (a) 500 times, (b) 5000 times, (c) 10000 times and (d) 30000 times.

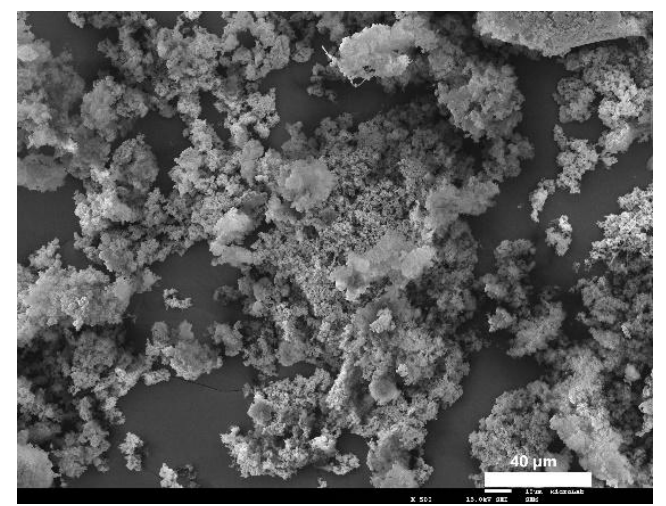

(a)

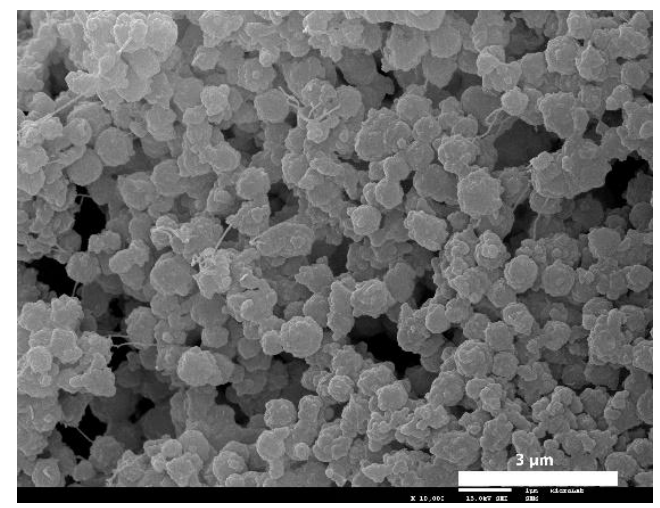

(c)

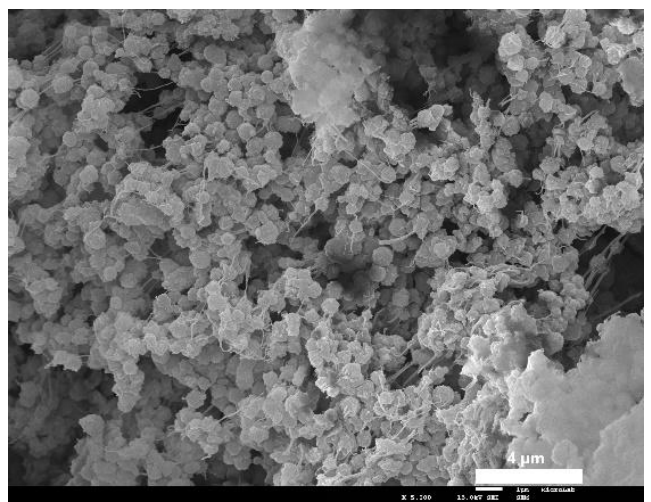

(b)

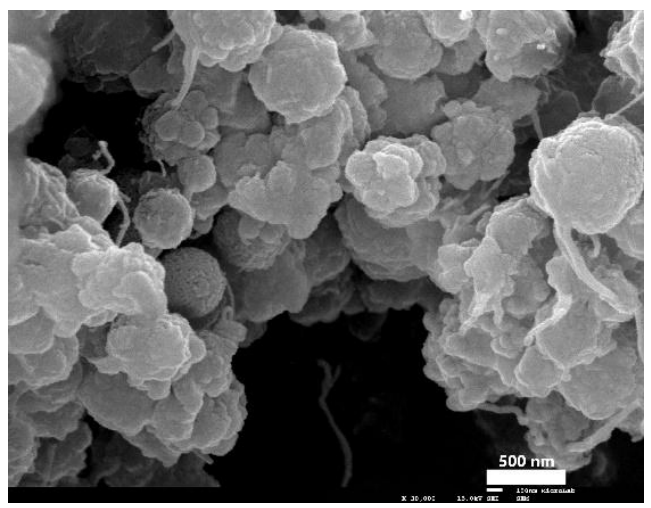

(d)

Figure 6 - SEM micrographs of polyethylene particles produced via the SA-DS method with different magnifications: (a) 500 times, (b) 5000 times, (c) 10000 times and (d) 30000 times.

Regardless of the magnification, the results show that polymers produced via the DSMAO method present a very fibrous morphology when compared to their SA-DS analogues. In industrial applications this fibrous morphology is not desirable, as support particle morphology replication is a crucial point in catalyst design. Previous publications from our research group by Campos et al. ${ }^{[50]}$ and Ferreira et al. ${ }^{[32]}$ showed a clear tendency for a fibrous morphology to develop when using metallocene systems supported on MCM-41 and SBA-15, enabling extrusion polymerization reactions inside the support's mesoporous channels. However, taking 
into account that the porosity of DS is inherently different to that of other types of silica, this behavior is not likely to present itself.

The morphology observed for the SA-DS polymers with more spherical polymer particles, replicating the initial shape of the support particles (see Figure 1), is more desirable in industrial terms. Similar morphologies to those observed in Figure 6 were reported by Lee and Park for polymers also prepared with in-situ supported catalytic systems ${ }^{[51]}$. It is interesting to observe that, in the case of DS-MAO, the fibrous morphology of the polymer promotes the formation of large agglomerates, contrary to what is observed for SA-DS. The long fibers that are formed in the former may possibly entangle with one another giving rise to the structures present in Figure 5 (a) and, consequently, the DS-MAO method appears to yield poorer polymer morphology control compared to the SA-DS method.

\section{Thermal characterization of the polymer powders}

In order to investigate the effect of the polymerization procedure on thermal properties the as-prepared reactor polymer powders were analyzed through differential scanning calorimetry. This is an important information to obtain with regards to the processability of the polymers. Table 3 presents the thermal characterization results in terms of crystalline fraction $\left(f_{c}\right)$, melting temperature $\left(T_{m}\right)$ and crystallization temperature $\left(T_{c}\right)$ obtained at first melting, crystallization and second melting transitions, for the samples previously reported.

Table 3 - DSC data for first melting (F1), crystallization (C) and second melting (F2) obtained for the polymer reactor powders prepared via the two different methods compared to polyethylene prepared with a homogeneous catalyst.

\begin{tabular}{|c|c|c|c|c|c|}
\hline Sample & $\left.\boldsymbol{T}_{\boldsymbol{m}}^{\boldsymbol{F 1}} \mathbf{(}^{\circ} \mathbf{C}\right)$ & $\boldsymbol{f}_{\boldsymbol{c}}^{\boldsymbol{F 1}}$ & $\left.\boldsymbol{T}_{\boldsymbol{m}}^{\boldsymbol{F 2}} \mathbf{(}^{\circ} \mathbf{C}\right)$ & $\boldsymbol{f}_{\boldsymbol{c}}^{\boldsymbol{F 2}}$ & $\left.\boldsymbol{T}_{\boldsymbol{c}} \mathbf{(}^{\circ} \mathbf{C}\right)$ \\
\hline HDPE & 136.0 & 0.77 & 135.0 & 0.57 & 114.8 \\
\hline DS-MAO & 138.6 & 0.76 & 136.8 & 0.54 & 116.0 \\
\hline SA-DS-Al/Zr470 & 135.0 & 0.72 & 133.8 & 0.59 & 118.6 \\
\hline SA-DS-Al/Zr320 & 137.7 & 0.83 & 136.9 & 0.58 & 116.6 \\
\hline SA-DS-Al/Zr160 & 138.6 & 0.81 & 136.5 & 0.57 & 117.5 \\
\hline SA-DS-Al/Zr80 & 139.3 & 0.74 & 136.0 & 0.59 & 118.2 \\
\hline
\end{tabular}


The thermal analysis of the polymers shows that the melting temperature in the first heating cycle of the polymer SA-DS-Al/Zr470 is slightly lower than the one observed for the reference HDPE obtained via polymerization with a homogeneous catalyst, while all the other samples show higher values. The increase in melting temperature is in agreement with the increase in weight average molar mass. In fact, samples SA-DS-AI/Zr80 and DS-MAO show quite high molar masses ( $M_{w}$ of 423000 and $515000 \mathrm{~g} / \mathrm{mol}$, respectively) and a $T_{m}^{F 1}$ of around $139{ }^{\circ} \mathrm{C}$, which is close to the theoretical maximum for HDPE. The unusually high melting temperatures observed suggest that disentangled HDPE chains are being generated under the employed polymerization conditions. The rather high values of $f_{c}^{F 1}$ displayed by all analyzed samples, in the range of 0.72 to 0.83 , also support this assumption.

At diluted catalyst and/or cocatalyst concentrations combined with low polymerization temperatures, the polymerization rate is lowered relative to the crystallization rate, which promotes the formation of disentangled chains during the reaction and enables a controlled oriented crystallization process of extended chain polymers. The larger crystallites formed during the process naturally take longer to melt, which causes the observable melting temperature to increase $\mathrm{e}^{[19,32,52,53]}$.

Franceschini et al. ${ }^{[54]}$ observed in a previously mentioned study that the polyethylene produced through in-situ supported systems (SA-DS equivalent) presented similar melting temperatures to the polymer produced with the catalyst in homogeneous conditions, while other studies published by Lee and Park ${ }^{[38,51]}$ reported polymers produced through in-situ supported systems that presented both higher and lower melting temperatures than their homogeneous analogues. In fact, there are various factors that contribute towards the final melting temperature and crystallinity of a polymer, namely the polymerization temperature, the type of system applied (catalyst, co-catalyst, scavenger, etc.). Thus, it is possible to modify the polymerization conditions to tune the thermal properties of the final polymer. 


\section{Conclusions}

In this work, an innovative ethylene polymerization procedure was explored with the goal of producing polyethylene nanocomposites, based on previously reported catalyst preparation methods referred to as in-situ catalyst supporting. Dubbed SA-DS, this procedure presents an advantage by reducing the number of steps required to yield the active catalytic system. An assessment of the SA-DS procedure showed highly active catalytic systems for the polymerization of ethylene while employing significantly lower amounts of MAO compared to more traditional methods, herein represented by the DS-MAO method. An analysis of the kinetic profiles showed that the SA-DS method presents a decay-type kinetic profile, similar to that exhibited by homogeneous systems, and opposed to the DS-MAO method, which exhibited a build-up type of kinetic profile. Additionally, it was found that by varying the amount of supported activator introduced during the SA-DS procedure, it is possible to achieve remarkable catalytic activities similar to those exhibited by the homogeneous system, as well as modify the deactivation rate during polymerization. An analysis of molar mass in the SA-DS polymers reveals a decreasing average molar mass when increasing the amount of supported activator employed. Comparing the two polymerization procedures, the SA-DS yielded lower average molar mass than the DS-MAO procedure, and closer to the values exhibited by the reference HDPE, obtained by solution polymerization.

A morphology assessment of the polymer powders prepared by the two different techniques showed systems with very different morphologies, those prepared through the DSMAO procedure presenting a more fibrous aspect and the DS particles interconnected with longer polymer nanofibers. The SA-DS procedure, on the other hand, yielded polymers with a more well-defined spherical morphology.

A subsequent thermal analysis by DSC showed polymer powders with very high crystallinity regardless of the preparation method. The SA-DS procedure was able to produce polymers with upwards of $80 \%$ crystallinity and a melting temperature close to the theoretical maximum of HDPE, indicating the formation of disentangled polymer chains during polymerization.

Overall, the in-situ polymerization procedure reported here shows promising results not only in terms of catalytic activity, but also the versatility it confers in tailoring polymer properties through the experimental conditions. Its application in the preparation of HDPE nanocomposites could show promise in the generation of highly performant nanocomposite materials for several applications and will be explored in further studies. 


\section{References}

[1] J. M. Campos, J. P. Lourenço, H. Cramail, M. R. Ribeiro, Prog. Polym. Sci. 2012, 37, 1764-1804.

[2] W. Kaminsky, A. Funck, K. Wiemann, Macromol. Symp. 2006, 239, 1-6.

[3] L. Wei, T. Tang, B. Huang, J. Polym. Sci. Part A Polym. Chem. 2004, 42, 941-949.

[4] K.-J. Chu, J. B. P. Soares, A. Penlidis, J. Polym. Sci. Part A Polym. Chem. 2000, 38, 462468.

[5] K. Ikenaga, S. Chen, M. Ohshima, H. Kurokawa, H. Miura, Catal. Commun. 2007, 8, 3638.

[6] M. de Fátima V. Marques, M. de Alcantara, J. Polym. Sci. Part A Polym. Chem. 2004, 42, 9-21.

[7] H. Xu, C.-Y. Guo, Eur. Polym. J. 2015, 65, 15-32.

[8] C. Favero, M. L. Mignoni, R. F. de Souza, K. Bernardo-Gusmão, J. Brazilian Chem. Soc. 2015, 26, 1405-1410.

[9] V. M. Maria de Fátima, L. F. M. Rocha, A. R. dos Santos, Macromol. Symp. 2019, 383, 1800001.

[10] M. W. Holtcamp, G. S. Day, L. G. McCullough, D. F. Sanders, Polymerization Process Using Bridged Metallocene Compounds Supported on Organoaluminum Treated Layered Silicate Supports, 2019, US10351647B2.

[11] A. Conte, M. F. . Marques, Eur. Polym. J. 2001, 37, 1887-1893.

[12] M. Smit, J. R. Severn, X. Zheng, J. Loos, J. C. Chadwick, J. Appl. Polym. Sci. 2006, 99, 986993.

[13] A. F. R. Kilpatrick, J.-C. Buffet, P. Nørby, N. H. Rees, N. P. Funnell, S. Sripothongnak, D. O'Hare, Chem. Mater. 2016, 28, 7444-7450.

[14] W. Kaminsky, Process for Producing Starch/Polyolefin Polymer Compositions, 1982, US4431788A.

[15] D. Lee, K. Yoon, Macromol. Rapid Commun. 1994, 15, 841-843.

[16] C. Yong, Z. Ning, L. Zi-Long, S. Wen-Hua, Chinese J. Chem. 2003, 21, 491-493.

[17] X. Wang, C. Zhang, W. Liu, P. Zhang, Polym. 2018, 10, DOI 10.3390/polym10090944.

[18] L. Wu, S. Wanke, Handb. Transit. Met. Polym. Catal. 2018, 369-400.

[19] A. E. Ferreira, M. L. Cerrada, E. Perez, V. Lorenzo, E. Vallés, J. Ressia, H. Cramail, J. Lourenço, M. Ribeiro, UHMWPE/HDPE in-Reactor Blends, Prepared by in Situ Polymerization: Synthetic Aspects and Characterization, 2017.

[20] M. L. Cerrada, A. Bento, E. Pérez, V. Lorenzo, J. P. Lourenço, M. R. Ribeiro, Microporous Mesoporous Mater. 2016, 232, 86-96.

[21] M. L. Cerrada, E. Pérez, J. P. Lourenço, A. Bento, M. R. Ribeiro, Polymer (Guildf). 2013, 
$54,2611-2620$.

[22] H. Zou, S. Wu, J. Shen, Chem. Rev. 2008, 108, 3893-3957.

[23] V. Polshettiwar, D. Cha, X. Zhang, J. M. Basset, Angew. Chemie - Int. Ed. 2010, 49, 96529656.

[24] S. Lee, K. Y. Choi, Macromol. React. Eng. 2017, 11, 1600027.

[25] D. M. Cecílio, A. Fernandes, J. P. Lourenço, M. R. Ribeiro, ChemCatChem 2018, O, DOI $10.1002 /$ cctc. 201800534 .

[26] K.-J. Chu, J. B. P. Soares, A. Penlidis, J. Polym. Sci. Part A Polym. Chem. 2000, 38, 18031810.

[27] S. Ahmadjo, H. Arabi, G. hossein Zohuri, G. reza Nejabat, M. Omidvar, M. Ahmadi, M. mehdi Mortazavi, J. Pet. Sci. Technol. 2014, 4, 21-29.

[28] J. M. Campos, J. P. Lourenço, A. Fernandes, A. M. Rego, M. R. Ribeiro, J. Mol. Catal. A Chem. 2009, 310, 1-8.

[29] F. A. Quinn, L. Mandelkern, J. Am. Chem. Soc. 1958, 80, 3178-3182.

[30] B. Wunderlich, Macromolecular Physics, Elsevier, 1980.

[31] J. M. Campos, J. P. Lourenco, E. Perez, M. L. Cerrada, M. R. Ribeiro, J. Nanosci. Nanotechnol. 2009, 9, 3966-3974.

[32] A. E. Ferreira, M. L. Cerrada, E. Pérez, V. Lorenzo, H. Cramail, J. P. Lourenço, M. R. Ribeiro, Microporous Mesoporous Mater. 2016, 232, 13-25.

[33] X. Dong, L. Wang, G. Jiang, Z. Zhao, T. Sun, H. Yu, W. Wang, J. Mol. Catal. A Chem. 2005, 240, 239-244.

[34] F. Silveira, M. do C. M. Alves, F. C. Stedile, S. B. Pergher, A. Rigacci, J. H. Z. dos Santos, J. Mol. Catal. A Chem. 2009, 298, 40-50.

[35] F. Silveira, M. do C. M. Alves, F. C. Stedile, S. B. Pergher, J. H. Z. dos Santos, J. Mol. Catal. A Chem. 2010, 315, 213-220.

[36] H. S. Zijlstra, S. Harder, Eur. J. Inorg. Chem. 2015, 2015, 19-43.

[37] D. Coevoet, H. Cramail, A. Deffieux, Macromol. Chem. Phys. 1998, 199, 1451-1457.

[38] H.-W. Lee, S.-H. Ahn, Y.-H. Park, J. Mol. Catal. A Chem. 2003, 194, 19-28.

[39] T. Sano, H. Hagimoto, S. Sumiya, Y. Naito, Y. Oumi, T. Uozumi, K. Soga, Microporous Mesoporous Mater. 2001, 44-45, 557-564.

[40] P. Kumkaew, L. Wu, P. Praserthdam, S. E. Wanke, Polymer (Guildf). 2003, 44, 47914803.

[41] P. Kumkaew, S. E. Wanke, P. Praserthdam, C. Danumah, S. Kaliaguine, J. Appl. Polym. Sci. 2003, 87, 1161-1177.

[42] M. R. Ribeiro, A. Deffieux, M. F. Portela, Ind. Eng. Chem. Res. 1997, 36, 1224-1237.

[43] D. Bianchini, K. M. Bichinho, J. H. Z. dos Santos, Polymer (Guildf). 2002, 43, 2937-2943.

[44] M. E. Z. Velthoen, A. Muñoz-Murillo, A. Bouhmadi, M. Cecius, S. Diefenbach, B. M. Weckhuysen, Macromolecules 2018, 51, 343-355. 
[45] W. Kaminsky, F. Renner, Die Makromol. Chemie, Rapid Commun. 1993, 14, 239-243.

[46] J. H. Z. dos Santos, C. Krug, M. B. da Rosa, F. C. Stedile, J. Dupont, M. de Camargo Forte, J. Mol. Catal. A Chem. 1999, 139, 199-207.

[47] R. Brambilla, C. Radtke, F. C. Stedile, J. H. Z. Dos Santos, M. S. L. Miranda, Appl. Catal. A Gen. 2010, 382, 106-114.

[48] M. Bochmann, Organometallics 2010, 29, 4711-4740.

[49] E. Quintanilla, F. di Lena, P. Chen, Chem. Commun. 2006, 4309-4311.

[50] J. M. Campos, M. R. Ribeiro, J. P. Lourenço, A. Fernandes, J. Mol. Catal. A Chem. 2007, 277, 93-101.

[51] H.-W. Lee, Y.-H. Park, Catal. Today 2002, 74, 309-320.

[52] A. E. Ferreira, M. L. Cerrada, E. Pérez, V. Lorenzo, H. Cramail, J. P. Lourenço, R. Quijada, M. R. Ribeiro, Eur. Polym. J. 2016, 85, 298-312.

[53] M. Stürzel, A. Kurek, M. Anselm, T. Halbach, R. Mülhaupt, in (Ed.: W. Kaminsky), Springer Berlin Heidelberg, Berlin, Heidelberg, 2013, pp. 279-309.

[54] F. C. Franceschini, T. T. da R. Tavares, J. H. Z. dos Santos, M. L. Ferreira, J. B. P. Soares, Macromol. Mater. Eng. 2006, 291, 279-287. 